\title{
The fat of the matter
}

Amid an obesity epidemic, the approval of two new obesity drugs might revamp the clinical landscape of obesity treatment.

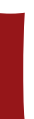
$\mathrm{n}$ spite of the growing burden of obesity worldwide, antiobesity drug development and approval have lagged behind. In the few cases in which a therapy has made the regulatory cut, as with sibutramine and rimonabant, safety concerns have forced regulators to pull the drugs from the market (see Nat. Med 18, 843, 2012). These failures over the past 13 years, and in particular the high bar for safety, have dampened the interest of pharmaceutical companies in this therapeutic space. But this situation may change after the approval of two new antiobesity drugs by the US Food and Drug Administration (FDA).

In June, the FDA approved lorcaserin (Belviq), a prescription weight-loss drug developed by Arena Pharmaceuticals that induces satiety through the activation of the $5 \mathrm{HT} 2 \mathrm{C}$ receptor, a subtype of serotonin receptors found only in the brain. In 2010, the FDA had rejected lorcaserin because of evidence that it increased cancer incidence in rats and because its modest efficacy did not outweigh safety concerns. New data presented by the company changed the views of the FDA advisory panel, which concluded that the cancer findings did not apply to humans.

Obese adults and overweight people with a concomitant weight-related comorbidity (type 2 diabetes, dyslipidemia or hypertension) are eligible for this drug. In two clinical studies carried out with nondiabetic individuals and one follow-up study with subjects with diabetes, lorcaserin led to an average loss of $3 \%$ body weight after one year of treatment when adjusted to placebo. Although this is less than the 5\% described as clinically significant by the FDA, the studies met other FDA criteria for approving weight-loss drugs-that at least $35 \%$ of treated subjects achieve $5 \%$ weight loss after one year and that this number is double the number of responders in the placebo group. In individuals with diabetes, lorcaserin also improved glycemic control, regardless of whether the patients lost weight.

The FDA's approval in July of Qsymia, developed by the small drug company Vivus, may signal a greater willingness of the FDA to tolerate certain side effects in an antiobesity drug. Qsymia is a combination of two older drugs, phentermine and topiramate, that reduces hunger by targeting different neurotransmitter systems, mainly norepinephrine but also dopamine and serotonin. Whereas this drug seems to induce more weight loss than lorcaserin in a similar target population (an average of $8.9 \%$ at the highest dose compared to placebo), it may cause more serious side effects, including an increased heart rate and increased risk of birth defects. Both Arena and Vivus have committed to carrying out post-marketing studies to assess adverse events. Given the numerous potential subjects and the required longterm treatment, these studies are crucial, as cardiovascular risk might already be higher in obese people.

Despite lingering concerns regarding potential side effects, the FDA was convinced that the benefits outweigh the risks, as there is evidence that even a $5 \%$ weight loss can have positive effects on blood pressure and metabolic syndrome. These drugs may help individuals achieve a consistent degree of weight loss, which may be crucial, as recidivism is common among dieters and a substantial barrier for obesity therapy. At the same time, patient education must clarify the drugs' real efficacy and expected outcome to avoid false expectations. According to the FDA, people should stop taking these drugs if they fail to lose $5 \%$ body weight in 12 weeks, something that happened in about $60 \%$ of the subjects taking lorcaserin during the clinical studies.

Lorcaserin's approval validates serotonin signaling as an antiobesity target and suggests that specifically targeting 5HT2C receptors could minimize undesired side effects. However, compensatory mechanisms affecting other neurotransmitter systems and circulating hormones that control weight might limit the drug's efficacy. A strategic use of drug combinations may provide synergistic effects by targeting different pathways to increase efficacy or avoid resistance. The development of drug combinations poses a challenge for drug companies and regulatory agencies, yet the complexity of the network regulating energy balance will probably require this multitarget strategy.

The FDA's decision to approve the drug despite its modest efficacy is a reflection of the magnitude of the obesity epidemic. In the US, $70 \%$ of adults are overweight and about $33 \%$ and $17 \%$ of adults and children, respectively, are obese. Although these numbers are now reaching a plateau in the US, the prevalence remains high, and, globally, obesity rates are predicted to keep on increasing. When considering this huge medical need and the lack of available drugs, it is not surprising that many within the medical community have applauded the approval of these two drugs.

Although Belbiq and Qsymia are not perfect drugs, and their use should not replace efforts to encourage long-term weight loss through a healthy lifestyle, they are an important addition to the antiobesity toolbox. These two drugs may be the harbinger of further pharmacological developments to help beat obesity by attacking it through different fronts. 